\title{
Robust Confidence Intervals for the Population Mean Alternatives to the Student- $t$ Confidence Interval
}

Moustafa Omar Ahmed Abu-Shawiesh

The Hashemite University, Zarqa, Jordan, mabushawiesh@hu.edu.jo

Aamir Saghir

Mirpur University of Science and Technology, Mirpur, Pakistan, aamirstat@yahoo.com

Follow this and additional works at: https://digitalcommons.wayne.edu/jmasm

Part of the Applied Statistics Commons, Social and Behavioral Sciences Commons, and the Statistical Theory Commons

\section{Recommended Citation}

Abu-Shawiesh, M. O. A., \& Saghir, A. (2019). Robust confidence intervals for the population mean alternatives to the Student- $t$ confidence interval. Journal of Modern Applied Statistical Methods, 18(1), eP2721. doi: 10.22237/jmasm/1556669160

This Regular Article is brought to you for free and open access by the Open Access Journals at DigitalCommons@WayneState. It has been accepted for inclusion in Journal of Modern Applied Statistical Methods by an authorized editor of DigitalCommons@WayneState. 


\section{Robust Confidence Intervals for the Population Mean Alternatives to the Student- $t$ Confidence Interval}

\section{Cover Page Footnote}

The authors are grateful to the Editor and anonymous three reviewers for their excellent and constructive comments/suggestions that greatly improved the presentation and quality of the article. This article was partially completed while the first author was on sabbatical leave (2014-2015) in Nizwa University, Sultanate of Oman. He is grateful to the Hashemite University for awarding him the sabbatical leave which gave him excellent research facilities. 


\title{
Robust Confidence Intervals for the Population Mean Alternatives to the Student- $t$ Confidence Interval
}

\author{
Moustafa Omar Ahmed Abu-Shawiesh Aamir Saghir \\ The Hashemite University \\ Zarqa, Jordan \\ Mirpur University of Science and Technology \\ Mirpur, Pakistan
}

In this paper, three robust confidence intervals are proposed as alternatives to the Student- $t$ confidence interval. The performance of these intervals was compared through a simulation study shows that $Q_{n}-t$ confidence interval performs the best and it is as good as Student's- $t$ confidence interval. Real-life data was used for illustration and performing a comparison that support the findings obtained from the simulation study.

Keywords: Confidence interval, robust, absolute deviation, median, coverage probability, average width, skewness, kurtosis

\section{Introduction}

In statistical inference, the Student- $t$ distribution is used for drawing any inference about the population mean $(\mu)$ in case that the population standard deviation $(\sigma)$ is unknown. Suppose that the random sample $X_{1}, X_{2}, \ldots, X_{n}$ is drawn from the normal distribution with population mean $(\mu)$ and unknown population variance $\left(\sigma^{2}\right)$, that is $X_{1}, X_{2}, \ldots, X_{n} \sim \mathrm{N}\left(\mu, \sigma^{2}\right)$, then the $(1-\alpha) 100 \%$ Student- $t$ confidence interval (CI) for the population mean $(\mu)$ can be constructed as follows:

$$
\mathrm{CI}=\bar{X} \mp t_{\left(\frac{\alpha}{2}, n-1\right)} \frac{S}{\sqrt{n}},
$$

where

doi: 10.22237/jmasm/1556669160 | Accepted: January 8, 2019; Published: April 6, 2020.

Correspondence: Moustafa Omar Ahmed Abu-Shawiesh, mabushawiesh@hu.edu.jo 


\section{ABU-SHAWIESH \& SAGHIR}

$$
\bar{X}=n^{-1} \sum_{i=1}^{n} X_{i}
$$

is the sample mean,

$$
S=\sqrt{(n-1)^{-1} \sum_{i=1}^{n}\left(X_{i}-\bar{X}\right)^{2}}
$$

is the sample standard deviation and $t_{(\alpha / 2, n-1)}$ is the upper percentage point of the Student- $t$ distribution with $(n-1)$ degrees of freedom, i.e. $\mathrm{P}\left(\mathrm{t}>t_{(\alpha, n-1)}\right)=\alpha$ (AbuShawiesh et al., 2009; Bonett \& Seier, 2003). The Student- $t$ distribution was developed by William Gosset in 1908 as a more robust way of testing hypotheses specifically when sample sizes are below 30 (Student, 1908).

There are two issues associated with the Student- $t$ confidence interval (CI). Firstly, the Student- $t$ distribution is symmetric and based on normality assumption. Therefore, the $(1-\alpha) 100 \%$ confidence interval (CI) for the population mean $(\mu)$ is also based on the normality assumption. However, the normality assumption is not fulfilled in reality. In such situations, the Student- $t$ approach is not very robust as discussed by many authors including David (1998), Boos and Hughes-Oliver (2000), Kelley (2005), Wilcox (2005), Bonett and Seier (2006), Zuo (2010), Leys et al. (2013), and Desharnais et al. (2015). Previous researchers have found that the Student- $t$ distribution performs well for small samples sizes and asymmetric distributions in terms of the coverage probability $(\mathrm{CP})$ coming close to the nominal confidence coefficient although its average widths (AW) and variability were not as small as other confidence intervals (Zhou, et al., 2005; Shi \& Kibria, 2007; Wang, 2001). Different confidence intervals estimates can be used to improve the coverage probability (CP) when the data follows a skewed distribution. Secondly, the sample standard deviation $(S)$ is used in the construction of the Student- $t$ confidence interval. The estimator $S$ is very sensitive to outliers or/and deviation from the normality assumption. In this case, a robust scale estimator is required to develop a confidence interval (CI) for the population mean $(\mu)$. An estimator is said to be robust, if it is fully efficient or nearly so for an assumed distribution, but maintains high efficiency for plausible alternatives (Hampel, 1974; Tiku \& Akkaya, 2004). The robustness property can be study the breakdown point and the influence function of any estimator. Rousseeuw and Croux (1993) proposed two robust scale estimators, namely $S_{n}$ and $Q_{n}$, as alternatives to median absolute deviation from 


\section{ROBUST CONFIDENCE INTERVALS FOR POPULATION MEAN}

sample median (MAD). The two robust estimators will be introduced in a later section.

\section{Robust Scale Estimators}

In this section, the three robust scale estimators used in this paper will be introduced. Let $X_{1}, X_{2}, \ldots, X_{n}$ be a random sample of size $\mathrm{n}$ drawn from any parent distribution having mean $\mu$ and standard deviation $\sigma$. Then the median absolute deviation from the sample median $(M A D)$ is defined as follows:

$$
M A D=M D\left\{\left|X_{i}-M D\right|\right\} ; \quad i=1,2,3, \ldots, n
$$

where $M D$ is the sample median, which is very insensitive to outliers and has a maximal $50 \%$ breakdown point (Rousseeuw \& Croux,1993). The statistic $b M A D$ is an unbiased estimator of $\sigma$, where $b=1.4826$, as given by Rousseeuw and Croux (1993). Also, the sample median (MD) is more robust location estimator than the sample mean $(\bar{X})$. The median absolute deviation from the sample median $(M A D)$ has the highest breakdown point possible which is $50 \%$ and the influence function of it is bounded but not smooth. The MAD has $37 \%$ efficiency for normal distribution (Rousseeuw \& Croux, 1993). For the given random sample $X_{1}, X_{2}, \ldots$, $X_{n}$, the $S_{n}$ robust scale estimator can be defined as follows:

$$
S_{n}=M D_{i}\left\{M D_{j}\left|X_{i}-X_{j}\right|\right\} ; \quad i=1,2,3, \ldots, n ; j=1,2,3, \ldots, n
$$

The statistic $c S_{n}$ will be an unbiased estimator of $\sigma$, where $c=1.1926$ is a factor for consistency (Rousseeuw \& Croux, 1993). The important robustness properties for the $S_{n}$ estimator are it is also has the highest breakdown point possible which is $50 \%$ and the influence function of it is also bounded. The $S_{n}$ estimator produces $58.23 \%$ efficiency in case of normal distribution which is better than that of the median absolute deviation from the sample median (MAD) (Rousseeuw \& Croux, 1993).

Finally, for the given random sample $X_{1}, X_{2}, \ldots, X_{n}$, the $Q_{n}$ robust scale estimator can be defined as follows:

$$
Q_{n}=M D\left\{\left|X_{i}-X_{j}\right| ; i<j\right\}_{(g)} ; \quad i=1,2,3, \ldots, n ; j=1,2,3, \ldots, n
$$




\section{ABU-SHAWIESH \& SAGHIR}

where

$$
g=\left(\begin{array}{l}
h \\
2
\end{array}\right)=\frac{h(h-1)}{2} \quad \text { and } \quad h=\left[\frac{n}{2}\right]+1 .
$$

The statistic $d Q_{n}$ is an unbiased estimator for $\sigma$, where the factor $d=2.2219$ is for consistency (Rousseeuw \& Croux, 1993). The $Q_{n}$ estimator has the highest breakdown point $50 \%$ and the influence function of it is smooth, bounded and has no discrete part. The $Q_{n}$ estimator has $82 \%$ efficiency which is better than that of MAD and $S_{n}$ estimators. However, the $S_{n}$ estimator performs better than the $Q_{n}$ estimator for small sample sizes (Rousseeuw \& Croux, 1993).

The robustness of the confidence interval has been studied by many researchers; see for example, Abu-Shawiesh et al. (2009) and Rothe (2017). The current article develops confidence intervals for the population mean $(\mu)$ when the population standard deviation $(\sigma)$ is unknown based on the above three robust scale estimators $\left(M A D, S_{n}\right.$, and $\left.Q_{n}\right)$. These modified robust confidence intervals are named as $M A D-t, S_{n}-t$ and $Q_{n}-t$, and they will handle symmetric distributions with kurtosis slightly lower, moderate or a little higher than the normal distribution. The exact distribution of robust estimators $\left(M A D, S_{n}\right.$, and $\left.Q_{n}\right)$ of scale is not available in the literature, thus, analytical comparison among these estimators could not be determined. Alternatively, an extensive simulation study is conducted to calculate the coverage probabilities and average widths for comparison across confidence intervals. The smaller widths indicate a better confidence interval when coverage probabilities are the same; on the other hand, higher coverage probabilities indicate a better confidence interval when widths are the same.

\section{Methodology}

Let $X_{1}, X_{2}, \ldots, X_{n}$ be a random sample of size $n$ drawn from any parent distribution having mean $\mu$ and standard deviation $\sigma$, then in this section we will derive and introduce the proposed confidence intervals for the population mean $(\mu)$ when the population standard deviation $(\sigma)$ is unknown based on the scale robust estimators $M A D, S_{n}$, and $Q_{n}$. The proposed robust confidence intervals are named as MAD- $t$, $S_{n-t}$, and $Q_{n}-t$. 


\section{ROBUST CONFIDENCE INTERVALS FOR POPULATION MEAN}

\section{The MAD- $t$ Confidence Interval}

The $(1-\alpha) 100 \%$ MAD- $t$ confidence interval for the population mean $(\mu)$ which is a modification of the classical Student- $t$ confidence interval can be constructed using the $M A D$ estimator as follows:

$$
\mathrm{CI}=M D \pm t_{\left(\frac{\alpha}{2}, n-1\right)} \frac{b M A D}{\sqrt{n}} .
$$

\section{The $S_{n} t$ Confidence Interval}

The $(1-\alpha) 100 \% S_{n}-t$ confidence interval for the population mean $(\mu)$ which is a modification of the classical Student- $t$ confidence interval can be constructed using the $S_{n}$ estimator as follows:

$$
\mathrm{CI}=M D \pm t_{\left(\frac{\alpha}{2}, n-1\right)} \frac{c S_{n}}{\sqrt{n}} .
$$

\section{The $Q_{n}-t$ Confidence Interval}

The $(1-\alpha) 100 \% Q_{n}-t$ confidence interval for the population mean $(\mu)$ which is a modification of the classical Student- $t$ confidence interval can be constructed using the $Q_{n}$ estimator as follows:

$$
\mathrm{CI}=M D \pm t_{\left(\frac{\alpha}{2}, n-1\right)} \frac{d Q_{n}}{\sqrt{n}}
$$

\section{Simulation Results}

In this section, the efficiency of the proposed three robust confidence intervals for the population mean $(\mu)$ is illustrated and compared with the existing Student- $t$ confidence interval via a Monte Carlo simulation study. All simulations were performed using programs written in the $\mathrm{R}$ statistical software for windows. The main aim of this simulation is to study the effect of the non-normality on the four confidence intervals based on several non-normal distributions. According to the literature survey, the coverage probability $(\mathrm{CP})$ and the average width (AW) of any confidence interval (CI) are used as evaluation criteria. The following two definitions provide the efficiency comparison criterions in this work: 


\section{ABU-SHAWIESH \& SAGHIR}

Definition (1). The coverage probability (CP) associated with a confidence interval $\mathrm{CI}=(\mathrm{L}(X), \mathrm{U}(X))$ for the unknown parameter $\theta$ is measured by $\mathrm{P}_{\theta}\{\theta \in(\mathrm{L}(X), \mathrm{U}(X))\}$; see Mukhopadhyay (2000).

Definition (2). The average width (AW) of a confidence interval, is simply the average (expected width) for the difference between the upper endpoint $\mathrm{U}(X)$ and the lower endpoint $\mathrm{L}(X)$ of a confidence interval $\mathrm{CI}=(\mathrm{L}(X), \mathrm{U}(X))$; see Barker (2002).

Different levels of confidence coefficient are used to find the required confidence intervals. Among these, 95\% confidence coefficient $(\alpha=0.05)$ is usually used in the literature. It is expected that the coverage probability $(\mathrm{CP})$ of any confidence interval $(\mathrm{CI})$ will be around $(1-\alpha)=0.95$ when the data follows a symmetric distribution (or $\mathrm{n}$ is sufficiently large). Actually, much deviation of the coverage probability $(\mathrm{CP})$ from the $(1-\alpha) 100 \%$ results into the less efficiency of the confidence interval (CI). Secondly, a shorter width (difference between $\mathrm{U}$ and L) provides a better confidence interval (CI). A method is considered to be more efficient than the other if it has the smaller width when both methods have the same coverage probabilities.

There were 50,000 simulation replications for each one of the following sample sizes: $10,25,50,75$, and 100 . We obtain the $(1-\alpha) 100 \%$ confidence interval denoted by $\mathrm{CI}=(\mathrm{L}, \mathrm{U})$ based on the 50,000 replicates and estimated the coverage probability $(\mathrm{CP})$ and the average width (AW), respectively, by using the following two formulas:

$$
\mathrm{CP}=\frac{\#(L \leq \mu \leq U)}{50000} \text { and } \mathrm{AW}=\frac{\sum_{i=1}^{50000}\left(U_{i}-L_{i}\right)}{50000}
$$

The simulated data are generated from the different parent distributions which will be listed later. The coverage probability (CP) and the average width (AW) are used as performance measures for the proposed robust confidence intervals. The performance of the proposed methods has also been compared with the performance of existing Student- $t$ confidence interval. The following simulation procedure is adopted here:

Step 1. A random sample of size 10, 25, 50, 75 and 100 is drawn from any parent distribution listed below. 


\section{ROBUST CONFIDENCE INTERVALS FOR POPULATION MEAN}

Step 2. The sample median and the estimate of the standard deviation using $S, M A D, S_{n}$, and $Q_{n}$ are calculated for the sample.

Step 3. The $(1-\alpha) 100 \%$ confidence interval based on the estimates determined in Step 2 is calculated.

Step 4. The width and coverage probability of confidence interval obtained in Step 3 are calculated for each estimate.

Step 5. The Steps 1 to 4 are repeated 50,000 times and the average width (AW) and coverage probability (CP) are reported in this work.

The lists of distributions (symmetric and skewed distributions with low, moderate and high kurtosis) that will be considered in this paper are:

1 The standard normal distribution.

2 The uniform distribution $(0,1)$.

3 The Beta $(2,2)$ distribution.

4 The Student- $t$ distribution having 5, 8 and 10 degrees of freedom $(d f)$.

5 The Logistic $(0,1)$ distribution.

6 The Laplace $(0,1)$ distribution.

7 The location contaminated normal with $\alpha \%$ contamination, that is: $(1-\alpha) \mathrm{N}\left(\mu_{1}, \sigma\right)+\alpha \mathrm{N}\left(\mu_{2}, \sigma\right)$, where $\alpha=0.2,0.1, \mu_{1}=0$, and $\mu_{2}=3,5,7$ will be considered.

8 The Gamma $(\alpha, 1)$ for $\alpha$ equals 2,3 and 5. The case $\alpha=1$ is the exponential distribution.

The mean, standard deviation, skewness, and kurtosis for the above selected list of distributions are displayed in Table 1. The distributions under consideration are classified according to their skewness and kurtosis into the following classes (see Table 1):

1 Normal distribution.

2 Symmetric with kurtosis less than that of normal distribution.

3 Symmetric with kurtosis slightly higher than that of normal distribution.

4 Symmetric with moderate and high kurtosis than that of normal distribution.

$5 \quad$ Skewed with low kurtosis.

6 Skewed with moderate to high kurtosis. 


\section{ABU-SHAWIESH \& SAGHIR}

Table 1. Different characteristics of the under considered distributions

\begin{tabular}{rrrrr} 
Distribution & Mean & Standard & Skewness & Kurtosis \\
\hline Normal $(0,1)$ & 0.00 & 1.0000 & 0.0000 & 3.00 \\
Uniform $(0,1)$ & 0.50 & 0.2890 & 0.0000 & 1.80 \\
Beta $(2,2)$ & 0.50 & 0.2240 & 0.0000 & 2.14 \\
$\mathrm{t}(5)$ & 0.00 & 1.2910 & 0.0000 & 9.00 \\
$\mathrm{t}(8)$ & 0.00 & 1.1550 & 0.0000 & 4.50 \\
$\mathrm{t}(10)$ & 0.00 & 1.1180 & 0.0000 & 4.00 \\
Logistic $(0,1)$ & 0.00 & 1.8140 & 0.0000 & 4.20 \\
Laplace $(0,1)$ & 0.00 & 1.4140 & 0.0000 & 6.00 \\
LC $(0.05,3)$ & 0.05 & 1.1950 & 0.6800 & 4.35 \\
Gamma (2, 1) & 2.00 & 1.4140 & 1.4140 & 6.00 \\
Gamma (3, 1) & 3.00 & 1.7320 & 1.1550 & 5.00 \\
Gamma (5, 1) & 5.00 & 2.2360 & 0.8940 & 4.20 \\
Exponential (1) & 1.00 & 1.0000 & 2.0000 & 9.00 \\
\hline
\end{tabular}

Table 2. Coverage probability $(C P)$ and average width (AW) for $N(0,1)$ distribution

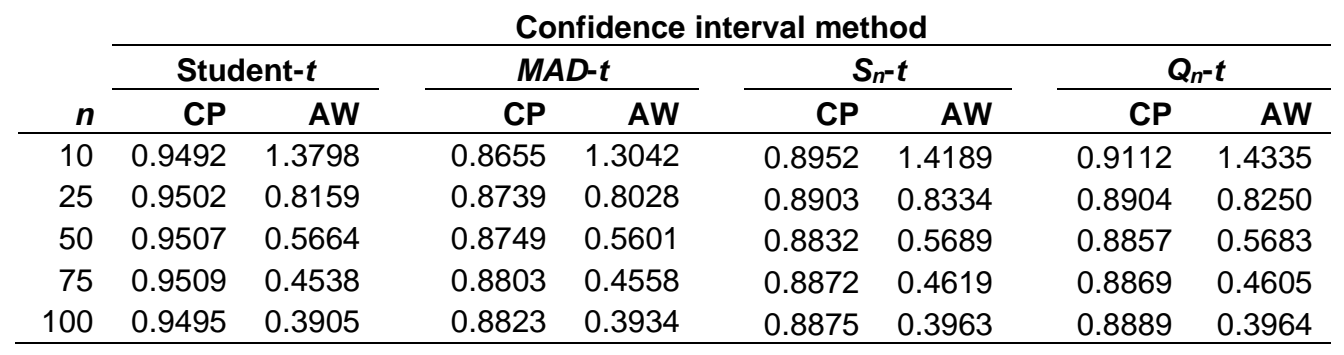

The coverage probability (CP) and the average width (AW) for the four methods of confidence interval estimation considered in this study for all various distributions are discussed below. The simulation results for the study are shown in Table 2 to Table 14.

\section{Symmetric Normal Distribution}

The efficiency of the four confidence intervals considered in this work is examined for the normal distribution and reported in Table 2.

The efficiency of all the compared CI's is almost same when data follows a normal distribution as it is clear from the results of Table 2. The coverage probability $(\mathrm{CP})$ is approximately $95 \%$, the same as the nominal value, for the four methods. The average widths (AW) for the four methods are about equal. It is in all confidence intervals decreases with the increasing of the sample sizes. 


\section{ROBUST CONFIDENCE INTERVALS FOR POPULATION MEAN}

\section{Symmetric with Kurtosis Less Than that of Normal Distribution}

This class of distributions includes the Uniform $(0,1)$ and Beta $(2,2)$ distributions.

As it can be seen from Table 3 and Table 4, the coverage probability (CP) for these two distributions ranges from $94.66 \%$ to $95.10 \%$ for the Student- $t, 90.90 \%$ to $97.87 \%$ for the $M A D-t, 92.18 \%$ to $97.26 \%$ for $S_{n}-t$, and $92.48 \%$ to $96.08 \%$ for the $Q_{n}-t$ based confidence intervals. The coverage probability (CP) increased with increasing sample size. For large samples the coverage probability $(\mathrm{CP})$ for Student- $t, M A D-t$ and $S_{n}-t$ is larger than that for the nominal coverage probability (CP), whereas for the $Q_{n}-t$ method, it is smaller for small samples and about the same for moderate and large samples. The $Q_{n}-t$ method perform better than the $M A D-t$ and $S_{n^{-}} t$ methods and approximately the same as the classical Student $t$ method especially for moderate and large sample sizes. The average width (AW) for this class of distributions is shorter than that for the normal distribution. The smallest average width for the three robust methods is achieved in the case of $Q_{n}-t$ method.

Table 3. Coverage probability $(\mathrm{CP})$ and average width $(\mathrm{AW})$ for $U(0,1)$ distribution

\begin{tabular}{|c|c|c|c|c|c|c|c|c|}
\hline \multirow[b]{3}{*}{$\boldsymbol{n}$} & \multicolumn{8}{|c|}{ Confidence interval method } \\
\hline & \multicolumn{2}{|c|}{ Student- $t$} & \multicolumn{2}{|c|}{ MAD-t } & \multicolumn{2}{|c|}{$S_{n-t}$} & \multicolumn{2}{|c|}{$Q_{n}-t$} \\
\hline & CP & AW & CP & AW & CP & AW & CP & AW \\
\hline 10 & 0.9467 & 0.4082 & 0.9090 & 0.4360 & 0.9218 & 0.4555 & 0.9248 & 0.4197 \\
\hline 25 & 0.9495 & 0.2376 & 0.9528 & 0.2831 & 0.9573 & 0.2701 & 0.9385 & 0.2424 \\
\hline 50 & 0.9507 & 0.1637 & 0.9720 & 0.2024 & 0.9689 & 0.1863 & 0.9513 & 0.1683 \\
\hline 75 & 0.9502 & 0.1326 & 0.9778 & 0.1659 & 0.9726 & 0.1497 & 0.9520 & 0.1362 \\
\hline 100 & 0.9510 & 0.1145 & 0.9787 & 0.1443 & 0.9699 & 0.1287 & 0.9502 & 0.1177 \\
\hline
\end{tabular}

Table 4. Coverage probability $(\mathrm{CP})$ and average width $(\mathrm{AW})$ for Beta $(2,2)$ distribution

\begin{tabular}{|c|c|c|c|c|c|c|c|c|}
\hline \multirow[b]{3}{*}{$n$} & \multicolumn{8}{|c|}{ Confidence interval method } \\
\hline & \multicolumn{2}{|c|}{ Student- $t$} & \multicolumn{2}{|c|}{ MAD-t } & \multicolumn{2}{|c|}{$S_{n-t}$} & \multicolumn{2}{|c|}{$Q_{n-t}$} \\
\hline & CP & AW & CP & AW & $\mathrm{CP}$ & AW & CP & AW \\
\hline 10 & 0.9442 & 0.3134 & 0.8806 & 0.3218 & 0.9304 & 0.3419 & 0.9377 & 0.3304 \\
\hline 25 & 0.9468 & 0.1833 & 0.8705 & 0.2012 & 0.9556 & 0.2008 & 0.9498 & 0.1926 \\
\hline 50 & 0.9503 & 0.1268 & 0.8655 & 0.1450 & 0.9660 & 0.1381 & 0.9608 & 0.1332 \\
\hline 75 & 0.9473 & 0.1025 & 0.8665 & 0.1189 & 0.9644 & 0.1115 & 0.9564 & 0.1077 \\
\hline 100 & 0.9490 & 0.0886 & 0.8733 & 0.1038 & 0.9635 & 0.0961 & 0.9582 & 0.0932 \\
\hline
\end{tabular}




\section{ABU-SHAWIESH \& SAGHIR}

Table 5. Coverage probability (CP) and average width (AW) for $t(8)$ distribution

\begin{tabular}{|c|c|c|c|c|c|c|c|c|}
\hline \multirow[b]{3}{*}{$n$} & \multicolumn{8}{|c|}{ Confidence interval method } \\
\hline & \multicolumn{2}{|c|}{ Student- $t$} & \multicolumn{2}{|c|}{$M A D-t$} & \multicolumn{2}{|c|}{$s_{n}-t$} & \multicolumn{2}{|c|}{$Q_{n}-t$} \\
\hline & CP & AW & CP & AW & CP & AW & CP & AW \\
\hline 10 & 0.9574 & 1.5895 & 0.8967 & 1.3922 & 0.9274 & 1.5310 & 0.9404 & 1.5699 \\
\hline 25 & 0.9545 & 0.9385 & 0.9108 & 0.8392 & 0.9318 & 0.8894 & 0.9379 & 0.8963 \\
\hline 50 & 0.9518 & 0.6503 & 0.9212 & 0.5887 & 0.9333 & 0.6093 & 0.9384 & 0.6175 \\
\hline 75 & 0.9490 & 0.5278 & 0.9169 & 0.4789 & 0.9273 & 0.4957 & 0.9320 & 0.4997 \\
\hline 100 & 0.9495 & 0.4567 & 0.9206 & 0.4121 & 0.9312 & 0.4245 & 0.9340 & 0.4302 \\
\hline
\end{tabular}

Table 6. Coverage probability (CP) and average width (AW) for $t(10)$ distribution

\begin{tabular}{|c|c|c|c|c|c|c|c|c|}
\hline \multirow[b]{3}{*}{$n$} & \multicolumn{8}{|c|}{ Confidence interval method } \\
\hline & \multicolumn{2}{|c|}{ Student- $t$} & \multicolumn{2}{|c|}{ MAD- $t$} & \multicolumn{2}{|c|}{$S_{n-t}$} & \multicolumn{2}{|c|}{$Q_{n}-t$} \\
\hline & CP & AW & CP & AW & CP & AW & CP & AW \\
\hline 10 & 0.9526 & 1.5418 & 0.8983 & 1.3704 & 0.9281 & 1.5041 & 0.9380 & 1.5346 \\
\hline 25 & 0.9528 & 0.9086 & 0.9163 & 0.8383 & 0.9404 & 0.8834 & 0.9436 & 0.8854 \\
\hline 50 & 0.9545 & 0.6309 & 0.9283 & 0.5834 & 0.9409 & 0.6015 & 0.9448 & 0.6082 \\
\hline 75 & 0.9494 & 0.5122 & 0.9234 & 0.4737 & 0.9333 & 0.4890 & 0.9381 & 0.4919 \\
\hline 100 & 0.9529 & 0.4417 & 0.9313 & 0.4087 & 0.9394 & 0.4188 & 0.9432 & 0.4230 \\
\hline
\end{tabular}

Table 7. Coverage Probability (CP) and average width (AW) for Logistic $(0,1)$ distribution

\begin{tabular}{|c|c|c|c|c|c|c|c|c|}
\hline \multirow[b]{3}{*}{$n$} & \multicolumn{8}{|c|}{ Confidence interval method } \\
\hline & \multicolumn{2}{|c|}{ Student- $t$} & \multicolumn{2}{|c|}{ MAD-t } & \multicolumn{2}{|c|}{$S_{n-t}$} & \multicolumn{2}{|c|}{$Q_{n}-t$} \\
\hline & CP & AW & CP & AW & CP & AW & CP & AW \\
\hline 10 & 0.9544 & 2.5047 & 0.8962 & 2.1921 & 0.9260 & 2.4121 & 0.9390 & 2.4679 \\
\hline 25 & 0.9530 & 1.4754 & 0.9093 & 1.3177 & 0.9320 & 1.3982 & 0.9372 & 1.4100 \\
\hline 50 & 0.9490 & 1.0230 & 0.9179 & 0.9164 & 0.9300 & 0.9519 & 0.9368 & 0.9677 \\
\hline 75 & 0.9533 & 0.8305 & 0.9216 & 0.7448 & 0.9345 & 0.7747 & 0.9387 & 0.7833 \\
\hline 100 & 0.9510 & 0.7173 & 0.9221 & 0.6439 & 0.9329 & 0.6650 & 0.9375 & 0.6747 \\
\hline
\end{tabular}

\section{Symmetric with Kurtosis Little More than Normal Distribution}

This class of distributions includes the $t(8), t(10)$ and Logistic $(0,1)$ distributions.

As it can be seen from Table 5, Table 6, and Table 7, the coverage probability (CP) for this class of distributions ranges from $94.94 \%$ to $95.74 \%$ for the Student- $t$, $89.62 \%$ to $93.13 \%$ for the $M A D-t, 92.60 \%$ to $94.09 \%$ for $S_{n}-t$, and $93.20 \%$ to $94.48 \%$ for the $Q_{n}-t$ based confidence intervals. In all intervals the coverage probability $(\mathrm{CP})$ decreasing with increasing sample sizes. Among the three robust methods, the closest coverage probability (CP) to the nominal is the $Q_{n}$ - $t$ interval. 


\section{ROBUST CONFIDENCE INTERVALS FOR POPULATION MEAN}

It is obviously clear that the $Q_{n}-t$ method perform better than the $M A D-t$ and $S_{n^{-}} t$ methods and approximately the same as the classical Student- $t$ method especially for moderate and large sample sizes. Regarding average width (AW) for this class of distributions, MAD-t interval is slightly shorter than that for the other three methods.

\section{Symmetric with Moderate to High Kurtosis Than that of Normal Distribution}

This class of distributions includes the $\mathrm{t}(5)$ and Laplace $(0,1)$ distributions.

As it can be seen from Table 8 and Table 9, the coverage probability (CP) for this class of distributions ranges from $94.86 \%$ to $95.61 \%$ for the Student- $t, 84.18 \%$ to $89.58 \%$ for the $M A D-t, 87.30 \%$ to $91.93 \%$ for $S_{n}-t$, and $88.92 \%$ to $93.29 \%$ for the $Q_{n}-t$ based confidence intervals. The results of the two tables show that the $Q_{n^{-}} t$ confidence interval is more robust than the other two robust methods. In all intervals, the coverage probability $(\mathrm{CP})$ increasing with increasing sample sizes. Among the three robust methods, the closest coverage probability (CP) to the nominal is the $Q_{n}-t$ interval. It is obviously clear that the $Q_{n}-t$ method perform better

Table 8. Coverage probability (CP) and average width (AW) for $t(5)$ distribution

\begin{tabular}{|c|c|c|c|c|c|c|c|c|}
\hline \multirow[b]{3}{*}{$n$} & \multicolumn{8}{|c|}{ Confidence interval method } \\
\hline & \multicolumn{2}{|c|}{ Student- $t$} & \multicolumn{2}{|c|}{ MAD-t } & \multicolumn{2}{|c|}{$s_{n-t}$} & \multicolumn{2}{|c|}{$Q_{n-t}$} \\
\hline & $\mathrm{CP}$ & AW & CP & AW & CP & AW & CP & AW \\
\hline 10 & 0.9539 & 1.7529 & 0.8821 & 1.4581 & 0.9167 & 1.6092 & 0.9329 & 1.6657 \\
\hline 25 & 0.9521 & 1.0364 & 0.8927 & 0.8704 & 0.9193 & 0.9335 & 0.9243 & 0.9442 \\
\hline 50 & 0.9514 & 0.7232 & 0.8958 & 0.6077 & 0.9119 & 0.6361 & 0.9230 & 0.6494 \\
\hline 75 & 0.9490 & 0.5884 & 0.8933 & 0.4931 & 0.9101 & 0.5162 & 0.9162 & 0.5243 \\
\hline 100 & 0.9486 & 0.5087 & 0.8905 & 0.4264 & 0.9067 & 0.4434 & 0.9143 & 0.4526 \\
\hline
\end{tabular}

Table 9. Coverage probability (CP) and average width (AW) for Laplace $(0,1)$ distribution

\begin{tabular}{|c|c|c|c|c|c|c|c|c|}
\hline \multirow[b]{3}{*}{$n$} & \multicolumn{8}{|c|}{ Confidence interval method } \\
\hline & \multicolumn{2}{|c|}{ Student- $t$} & \multicolumn{2}{|c|}{ MAD-t } & \multicolumn{2}{|c|}{$S_{n-t}$} & \multicolumn{2}{|c|}{$Q_{n-t}$} \\
\hline & $\mathrm{CP}$ & AW & $\mathbf{C P}$ & AW & $\mathbf{C P}$ & AW & $\mathbf{C P}$ & AW \\
\hline 10 & 0.9561 & 1.9068 & 0.8489 & 1.4554 & 0.8909 & 1.6353 & 0.9111 & 1.7308 \\
\hline 25 & 0.9532 & 1.1406 & 0.8421 & 0.8473 & 0.8833 & 0.9290 & 0.8971 & 0.9678 \\
\hline 50 & 0.9529 & 0.7930 & 0.8459 & 0.5832 & 0.8807 & 0.6256 & 0.8976 & 0.6601 \\
\hline 75 & 0.9515 & 0.6459 & 0.8418 & 0.4739 & 0.8730 & 0.5101 & 0.8892 & 0.5341 \\
\hline 100 & 0.9487 & 0.5569 & 0.8465 & 0.4073 & 0.8736 & 0.4349 & 0.8931 & 0.4583 \\
\hline
\end{tabular}




\section{ABU-SHAWIESH \& SAGHIR}

than the MAD-t and $S_{n}$ - $t$ methods and approximately the same as the classical Student- $t$ method especially for moderate and large sample sizes. Regarding average width (AW) for this class of distributions, $M A D$ - $t$ interval is slightly shorter than that for the other three methods.

\section{Skewed with Low Kurtosis}

This class of distributions includes the $\operatorname{LC}(0.05,3)$ and $\operatorname{Gamma}(5,1)$ distributions. As can be seen from Table 10 and Table 11, the coverage probability (CP) for this class of distributions ranges from $94.65 \%$ to $95.35 \%$ for the Student- $t, 88.72 \%$ to $94.53 \%$ for the MAD- $t, 91.57 \%$ to $95.27 \%$ for $S_{n}-t$, and $92.41 \%$ to $95.19 \%$ for the $Q_{n}-t$ based confidence intervals. The changes of coverage probability (CP) with sample sizes are minor. The coverage probability (CP) fluctuate with sample size changes. As far as average width concerned, the MAD- $t, S_{n}-t, Q_{n}-t$ have about the same width while the Student- $t$ has slightly longer average width (AW) especially in the case of Gamma $(5,1)$ distribution.

Table 10. Coverage probability (CP) and average width (AW) for $\operatorname{LC}(0.05,3)$ distribution

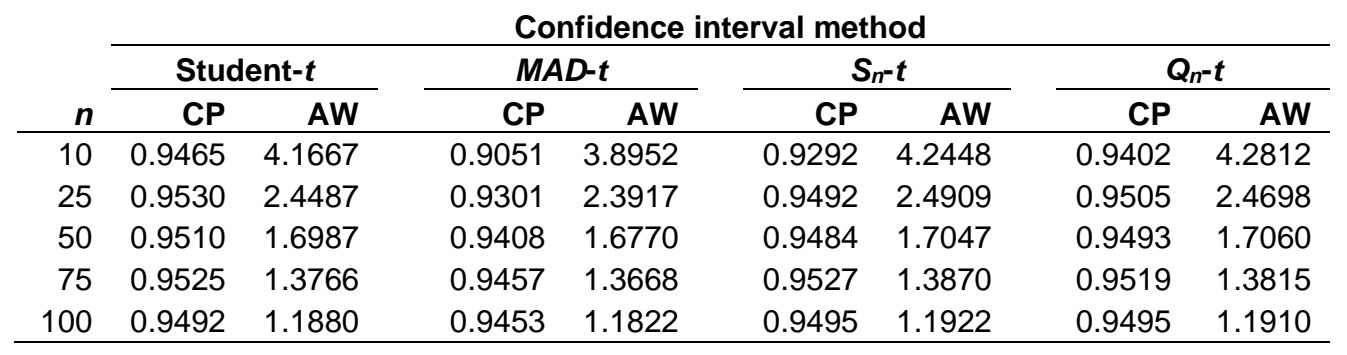

Table 11. Coverage probability (CP) and average width (AW) for Gamma $(5,1)$ distribution

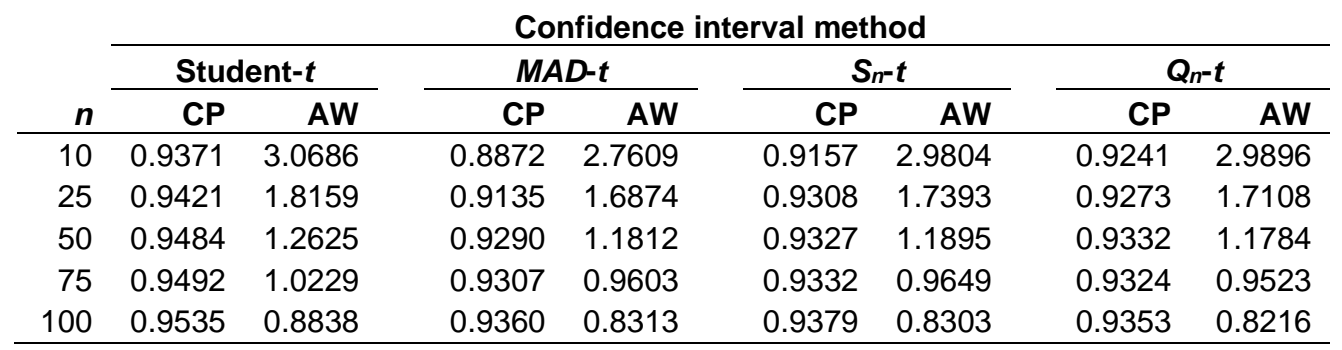




\section{ROBUST CONFIDENCE INTERVALS FOR POPULATION MEAN}

The results of the two tables show that the $Q_{n}-t$ confidence interval is more robust than the other two robust methods and has the closest coverage probability (CP) to the nominal. It is obviously clear that the $Q_{n}-t$ method perform better than the $M A D$ - $t$ and $S_{n}-t$ methods and approximately has the same as coverage probability (CP) as that of the Student- $t$ method.

\section{Skewed with Moderate to High Kurtosisy}

This class of distributions includes the Gamma $(2,1)$, Gamma $(3,1)$ and the Exponential (1) distributions. As it can be seen from Table 12, Table 13, and Table 14 , the coverage probability $(\mathrm{CP})$ for this class of distributions ranges from $89.85 \%$ to $94.83 \%$ for the Student- $t, 79.90 \%$ to $91.82 \%$ for the $M A D-t, 81.82 \%$ to $92.12 \%$ for $S_{n-t}$, and $79.31 \%$ to $91.76 \%$ for the $Q_{n}-t$ based confidence intervals.

The results of this class of distributions obviously show that the coverage probability $(\mathrm{CP})$ for the four methods diverts away from the nominal value. Among the robust methods the best coverage probability (CP) is for $S_{n}-t$ and $Q_{n}-t$ methods. And the shortest average width (AW) is for the MAD- $t$ method.

Table 12. Coverage probability (CP) and average width (AW) for Gamma $(2,1)$ distribution

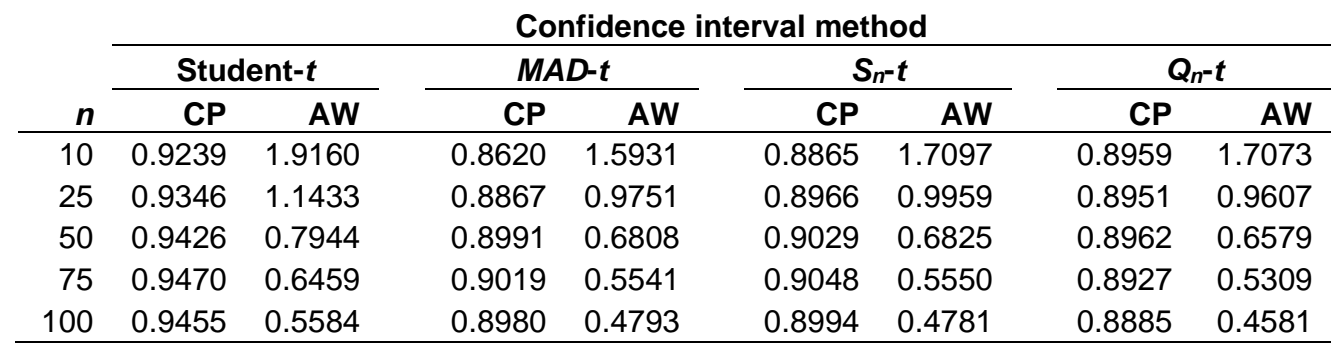

Table 13. Coverage probability (CP) and average width (AW) for Gamma $(3,1)$ distribution

\begin{tabular}{|c|c|c|c|c|c|c|c|c|}
\hline \multirow[b]{3}{*}{$n$} & \multicolumn{8}{|c|}{ Confidence interval method } \\
\hline & \multicolumn{2}{|c|}{ Student- $t$} & \multicolumn{2}{|c|}{ MAD- $t$} & \multicolumn{2}{|c|}{$S_{n}-t$} & \multicolumn{2}{|c|}{$Q_{n}-t$} \\
\hline & CP & AW & CP & AW & CP & AW & CP & AW \\
\hline 10 & 0.9333 & 2.3640 & 0.8799 & 2.0500 & 0.9051 & 2.2063 & 0.9143 & 2.2098 \\
\hline 25 & 0.9413 & 1.4060 & 0.8983 & 1.2570 & 0.9136 & 1.2914 & 0.9108 & 1.2588 \\
\hline 50 & 0.9467 & 0.9764 & 0.9174 & 0.8807 & 0.9201 & 0.8835 & 0.9176 & 0.8659 \\
\hline 75 & 0.9483 & 0.7909 & 0.9182 & 0.7153 & 0.9212 & 0.7163 & 0.9155 & 0.6981 \\
\hline 100 & 0.9450 & 0.6822 & 0.9149 & 0.6160 & 0.9153 & 0.6144 & 0.9109 & 0.6019 \\
\hline
\end{tabular}




\section{ABU-SHAWIESH \& SAGHIR}

Table 14. Coverage probability (CP) and average width (AW) for Exponential (1) distribution

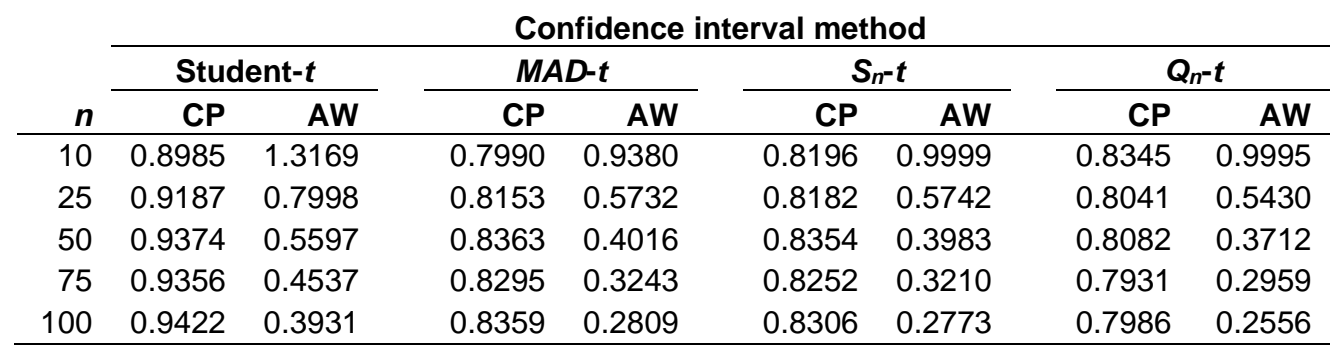

\section{Applications Using Real Data}

The proposed robust confidence intervals as well as the Student- $t$ confidence interval, are applied to two real-life data examples.

\section{Example 1 (Psychotropic Drug Exposure)}

To study the average use of psychotropic drugs from non-antipsychotic drug users, the number of users of psychotropic drugs was reported for twenty different categories of drugs; the following data represent the number of users (Johnson \& McFarland, 1993):

$\begin{array}{llllllllll}43.4 & 24 & 1.8 & 0 & 0.1 & 170.1 & 0.4 & 150 & 31.5 & 5.2 \\ 35.7 & 27.3 & 5 & 64.3 & 70 & 94 & 61.9 & 9.1 & 38.8 & 14.8\end{array}$

The objective is to calculate the average number of users of psychotropic drugs for non-antipsychotic drug users. The data is checked and found to be positively skewed data with skewness $=1.57$, kurtosis $=2.06$, mean $=42.37$ and standard deviation $=48.43$. A histogram of the data values showing its positive skewness is given in Figure 1. The considered confidence intervals and their corresponding widths have been given in Table 15.

From Table 15, observe the $S_{n}-t$ and $Q_{n}-t$ confidence intervals have the smallest width followed by $M A D-t$ confidence Interval. The Student- $t$ confidence interval has the largest width. Thus, the $S_{n}-t$ confidence interval performs the best among the compared confidence intervals as it produces smaller width. Those results are expected. 


\section{ROBUST CONFIDENCE INTERVALS FOR POPULATION MEAN}

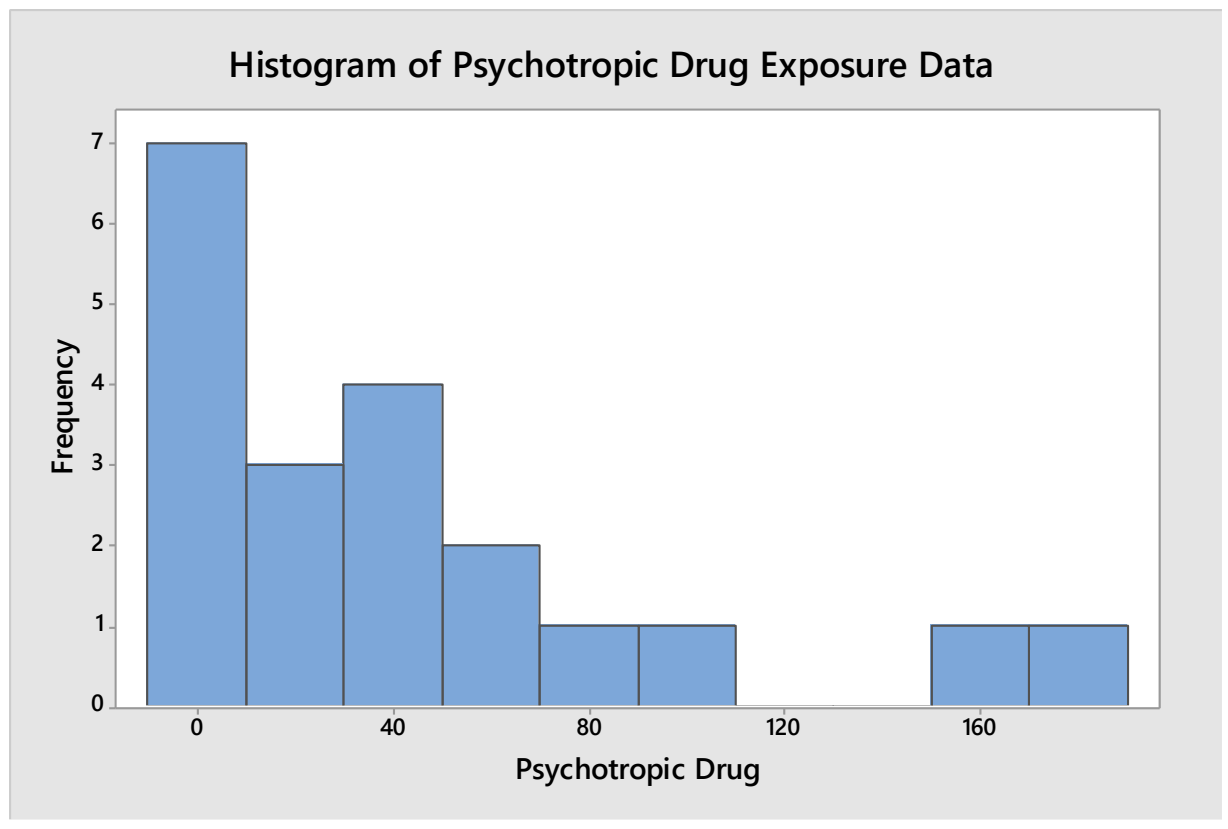

Figure 1. Histogram of psychotropic drug exposure data

Table 15. The 95\% confidence intervals for psychotropic drug exposure data

\begin{tabular}{rrr} 
Confidence interval method & Confidence interval & Width \\
\hline Student $t$ & $(19.704,65.036)$ & 45.333 \\
$M A D-t$ & $(24.329,60.411)$ & 36.082 \\
$S_{n}-t$ & $(25.011,59.728)$ & 34.717 \\
$Q_{n}-t$ & $(24.823,59.926)$ & 35.112 \\
\hline
\end{tabular}

\section{Example-2 (Long Jump Distance)}

The following data represent the results of the final points scores reported for 40 players in long jump distance in meters (International Olympic Committee, 2019):

$\begin{array}{llllllllll}8.11 & 8.11 & 8.09 & 8.08 & 8.06 & 8.03 & 8.02 & 7.99 & 7.99 & 7.97 \\ 7.95 & 7.92 & 7.92 & 7.92 & 7.89 & 7.87 & 7.84 & 7.79 & 7.79 & 7.77 \\ 7.76 & 7.72 & 7.71 & 7.66 & 7.62 & 7.61 & 7.59 & 7.55 & 7.53 & 7.50 \\ 7.50 & 7.42 & 7.38 & 7.38 & 7.26 & 7.25 & 7.08 & 6.96 & 6.84 & 6.55\end{array}$

The data are checked and found to be negatively skewed with skewness $=-1.16$, kurtosis $=1.20$, mean $=7.6745$, and standard deviation $=0.37$ and as it is also clear 


\section{ABU-SHAWIESH \& SAGHIR}

from Figure 2. Table 16 gives the confidence intervals and associated width of these. Table 16 shows the $Q_{n}$ - $t$ confidence interval has the smallest width followed by $M A D-t$ and $S_{n}-t$ confidence intervals. The classical Student- $t$ confidence interval has the largest width. Thus, the $Q_{n}-t$ confidence interval performs the best in the sense of having smaller width than the other confidence intervals. The results of this example supported the simulation study results.

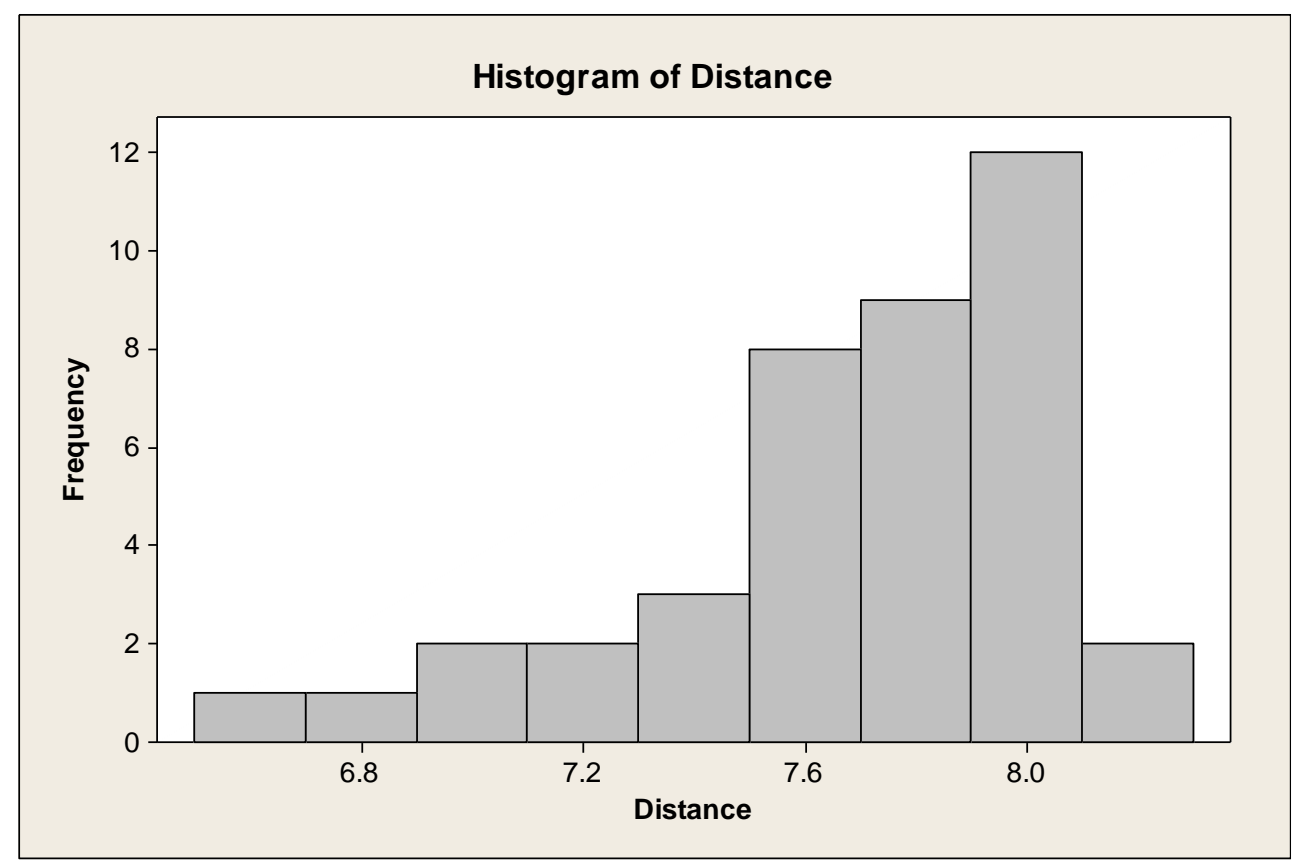

Figure 2. Histogram of long jump distance Olympic Games data

Table 16. The $95 \%$ confidence intervals for long jump distance data

\begin{tabular}{rrr} 
Confidence interval method & Confidence interval & Width \\
\hline Student $t$ & $(7.5562,7.7928)$ & 0.2366 \\
$M A D-t$ & $(7.5678,7.7812)$ & 0.2134 \\
$S_{n}-t$ & $(7.5600,7.7889)$ & 0.2288 \\
$Q_{n}-t$ & $(7.5706,7.7784)$ & 0.2077 \\
\hline
\end{tabular}

\section{Conclusion}

Three robust confidence intervals were proposed, namely $M A D-t, S_{n}-t$, and $Q_{n}-t$, as alternatives to the Student- $t$ confidence interval for estimating the mean of 


\section{ROBUST CONFIDENCE INTERVALS FOR POPULATION MEAN}

population $(\mu)$ when the population standard deviation $(\sigma)$ is unknown. The proposed methods, considered in this study, are sensitive to the moderate deviations from normality. Their coverage probability $(\mathrm{CP})$ going close to each other's when the sample size $n$ is sufficiently large. In particular, the methods prove robustness for samples from symmetric distributions with kurtosis slightly lower or slightly higher than that of the normal distribution. However, the $Q_{n}-t$ method proves the best coverage probability (CP) among the three robust confidence intervals. Also, it's coverage probability (CP) is very close to the nominal value $95 \%$ and to that of the exact Student- $t$ method in all sampled distributions. Therefore, it is recommended to use the findings of this work for the statistical inference regarding the population mean $(\mu)$ when the population standard deviation $(\sigma)$ is unknown.

\section{Acknowledgements}

The authors are deeply thankful to the editor and anonymous referees for their invaluable constructive comments and suggestions, which helped clarify several ideas and improved the quality and presentation of this paper.

\section{References}

Abu-Shawiesh, M. O., Al-Athari, F. M. \& Kittani, H. F. (2009). Confidence interval for the mean of a contaminated normal distribution. Journal of Applied Sciences, 9(15), 2835-2840. doi: 10.3923/jas.2009.2835.2840

Barker, L. (2002). A comparison of nine confidence intervals for a Poisson parameter when the expected number of events is $\leq 5$. The American Statistician, 56(2), 86-89. doi: 10.1198/000313002317572736

Bonett, D. G., \& Seier, E. (2003). Confidence intervals for mean absolute deviations. The American Statistician, 57(4), 233-236. doi:

10.1198/0003130032323

Bonett, D. G., \& Seier, E. (2006). Confidence intervals for a coefficient of dispersion in nonnormal distributions. Biometrical Journal, 48(1), 144-148. doi: 10.1002/bimj.200410148

Boos, D., \& Hughes-Oliver, J. (2000). How large does $n$ have to be for $Z$ and $t$ intervals. The American Statistician, 54(2), 121-128. doi: 10.1080/00031305.2000.10474524

David, H. (1998). Early sample measures of variability. Statistical Science, 13(4), 368-377. doi: 10.1214/ss/1028905831 


\section{ABU-SHAWIESH \& SAGHIR}

Desharnais, B., Lemyre, F. C., Mireault, P., \& Skinner, C. D. (2015). Determination of confidence intervals in nonnormal data: application of the bootstrap to cocaine concentration in femoral blood. Journal of Analytical Toxicology, 39(2), 113-117. doi: 10.1093/jat/bku127

Hampel, F. R. (1974). The influence curve and its role in robust estimation. Journal of the American Statistical Association, 69(346), 383-393. doi: 10.1080/01621459.1974.10482962

International Olympic Committee. (2019). London 2012 long jump men Olympic athletics. Retrieved from https://www.olympic.org/london2012/athletics/long-jump-men

Johnson, R. E., \& McFarland, B. H. (1993). Antipsychotic drug exposure in a health maintenance organization. Medical Care, 31(5), 432-444. doi: 10.1097/00005650-199305000-00005

Kelley, K. (2005). The effects of nonnormal distributions on confidence intervals around the standardized mean difference: bootstrap and parametric confidence intervals. Educational and Psychological Measurement, 65(1), 51-69. doi: 10.1177/0013164404264850

Leys, C., Ley, C., Klein, O., Bernard, P., \& Licata, L. (2013). Detecting outliers: not use standard deviation around the mean, use absolute deviation around the median. Journal of Experiment Social Psychology, 49(4), 764-766. doi: 10.1016/j.jesp.2013.03.013

Mukhopadhyay, N. (2000). Probability and statistical inference. New York: Marcel Dekker Inc.

Rothe, B. C. (2017). Robust confidence interval for average treatment effects under limited overlap. Econometrica, 85(2), 645-660. doi:

10.3982/ecta13141

Rousseeuw, P. J., \& Croux, C. (1993). Alternatives to the Median Absolute Deviation. Journal of the American Statistical Association, 88(424), 1273-1283. doi: 10.1080/01621459.1993.10476408

Shi, W. \& Kibria, B. M. G. (2007). On some confidence intervals for estimating the mean of a skewed population. International Journal of Mathematical Education and Technology, 38(3), 412-421. doi:

10.1080/00207390601116086

Student. (1908). The probable error of a mean. Biometrika, 6(1), 1-25. doi: $10.2307 / 2331554$ 


\section{ROBUST CONFIDENCE INTERVALS FOR POPULATION MEAN}

Tiku, M. L., \& Akkaya, A. D. (2004). Robust estimation and hypothesis testing. New Delhi, India: New Age International (P) Limited.

Wang, F. K. (2001). Confidence interval for a mean of non-normal data. Quality and Reliability Engineering International, 17(4), 257-267. doi:

10.1002/qre.400

Wilcox, R. R. (2005). Robust estimation and hypothesis testing. Burlington, MA: Elsevier Academic Press.

Zhou, X. H., \& Dinh, P. (2005). Nonparametric confidence intervals for the on and two-sample problems. Biostatistics, 6(2), 187-200. doi:

10.1093/biostatistics/kxi002

Zuo, Y. (2010). Is the $t$ confidence interval $\bar{X} \pm t_{\alpha}(n-1) s / \sqrt{ } n$ optimal? The American Statistician 64(2), 170-173. doi: 10.1198/tast.2010.09021 